\title{
An analytical approach to optimize radial synthetic aperture focusing for volumetric transrectal ultrasound imaging
}

\author{
Hyunwoo Song, Student Member, IEEE, Jeeun Kang, Member, IEEE, Emad M. Boctor, Senior Member, \\ IEEE
}

\begin{abstract}
In this paper, we present a novel analytical approach to optimize radial synthetic aperture focusing framework for high-definite and high-sensitive volumetric transrectal ultrasound imaging (TRUS-rSAF). A closed-form analytical description of beam profile defines spatial resolution and grating lobe positions in the TRUS-rSAF imaging of radial plane and validated by a heuristic testing of the critical parameters. Given the theoretical foundation, we optimize the TRUS-rSAF system configuration to balance the spatial and temporal resolution, grating lobe artifacts, and signal-to-noise ratio (SNR) in radial plane with a design criterion to outperform a clinical volumetric TRUS (TRUS-REF) imaging. The results showed that the proposed analytical optimization provides significant improvements of imaging quality in radial plane even over an in-plane microconvex TRUS imaging. Therefore, our analytical approach provides a optimal framework for effective TRUSrSAF imaging in clinics.
\end{abstract}

Index Terms-synthetic aperture focusing, transrectal ultrasound, analytical, optimization.

\section{INTRODUCTION}

$\mathrm{T}$ ransrectal ultrasound (TRUS) imaging is an effective clinical tool for cancer localization, biopsy guidance, and post-treatment surveillance [1], [2]. For several decades, 2dimensional (2-D) TRUS imaging using a 1-D linear or curved array transducer has been a standard protocol, but it has high dependency on the clinician's dexterity and subjective anatomic interpretation. Clinical urology reports its advantages of 3-D TRUS imaging, providing comprehensive anatomic context in prostate, renal and pelvic regions [3], in which volumetric scanning is implemented straightforwardly - Each radial plane is reconstructed with a single transmittance / reception event and repeats until filling the entire target volume using a motorized actuator [4]. However, the spatial acuity has been limited with a fixed lens focusing in a TRUS transducer [5]-[7].

Synthetic aperture focusing (SAF) techniques have been highlighted in the modern US imaging field for decades, which coherently compound time-multiplexed transmittance / reception events over sequential apertures at a specific target pixel, leading to higher spatial resolution along with enhanced texture uniformity [5]. Even though most of the prior arts have focused on developing better imaging quality in lateral direction [6]-[9], there have also been endeavors to effectuate the SAF technique in volumetric US imaging [10]-[15]. T. Lucas et al. presented a SAF technique to synthesize multiple cross-sections in different incident angles and positions in elevational dimension for higher spatial and contrast resolution [16]. However, it requires a sophisticated wobbling scanning in open imaging field-of-view (FOV), which is inapplicable to the TRUS imaging setup. On the other hand, intravascular US (IVUS) imaging was also tested with the SAF technology, hoping to break through the limitation in spatial resolution defined by rotational scanning interval and focusing tightness. This is a notably similar scenario what expected in radial scanning for the volumetric TRUS imaging. However, a recent investigation by $\mathrm{S}$. Kang et al. concluded that the SAF technique is not effective with the IVUS imaging configuration [17]. J. S. Kim et al. recently presented a radial SAF-enhanced framework with a customized TRUS transducer [18]. However, the progress has been stagnant primarily due to lack of an analytical approach that enables a theoretical optimization of the TRUS imaging framework.

In this paper, we present a radial synthetic aperture focusing framework for volumetric transrectal ultrasound imaging (TRUS-rSAF). To the best of our knowledge, we are the very first to establish a closed-form analytical approach optimizing the TRUS-rSAF imaging in radial direction (Section II). In Section III, critical parameters, defined from the analytical solution, were optimized to achieve the highest spatial resolution (Section III). The corresponding change of signal sensitivity and grating lobe artifacts were also characterized to balance the TRUS imaging quality. In Section IV, we present a practical case study how to balance between spatial and temporal resolution, grating lobe artifacts, and contrast with a design criterion to over-satisfy a clinical expectation given in a clinical TRUS (TRUS-REF) imaging.

\section{THEORY AND IMPLEMENTATION}

\section{A. Field analysis model of TRUS-rSAF imaging}

Fig. 1 illustrates the 2-D model of the TRUS-rSAF imaging framework. The $y$ - and $z$-axis represent elevational and axial directions of the linear TRUS array transducer, respectively. The acoustic source rotates along with the origin with a rotating radius $r=\sqrt{y_{o}^{2}+z_{0}^{2}}$. In the figure, the acoustic wave propagates along with a scanning angle $\theta=\sin ^{-1} \alpha$ with

H. Song and J. Kang contributed equally to the work. E. Boctor and J. Kang are co-corresponding authors. This work was supported by the Congressionally Directed Medical Research Programs, U.S. Department of Defense (DOD), USA (W81XWH-18-1-0188), NIH National Cancer Institute (NCI), USA (R01CA134675), and NSF Career award (1653322).

H. Song and E. M. Boctor are with the Computer Science, Johns Hopkins University (JHU) Whiting School of Engineering (WSE), MD 21218 USA. J. Kang and E. M. Boctor are with the Laboratory for Computational Sensing and Robotics, JHU WSE, MD 21218, USA. 
respect to the $z$-axis. Therefore, the velocity potential $\Phi$ of a monochromatic spherical wave at an observation point $(y, z)$ can be expressed as

$$
\Phi_{\alpha}(y, z, t)=e^{-j \omega t} \Psi_{\alpha}(y, z),
$$

where $\omega$ and $\Psi_{\alpha}$ represent the angular frequency of the transmitted acoustic wave and transmit beam pattern, respectively. The transmit beam pattern at a depth $R$ can be expressed as

$$
\Psi_{\alpha}(y, z)=e^{j k R}=e^{j k \sqrt{(y-r \alpha)^{2}+(z-r \beta)^{2}}},
$$

where $k=2 \pi / \lambda$ represents the wavenumber and $\beta=\cos \theta$.

In the defined coordination, the transmit beam is synthesized by compounding multiple acoustic waves propagating with different transmit angles by compensating corresponding synthetic time delays $\tau(\alpha)$ to be coherently focused on a desired focal point, $\left(y_{f}, z_{f}\right)$. Thus, the resultant beam pattern can be expressed as

$$
\Psi_{\mathrm{rSAF}}\left(y_{f}, z_{f}\right)=\int_{-\infty}^{\infty} p(\alpha) \tau(\alpha) \Psi_{\alpha}(y, z) d \alpha,
$$

where $\tau(\alpha)$ represents the synthetic time delay function and $p(\alpha)$ denotes the effective synthetic angular window over the range of $\alpha$ used in the TRUS-rSAF imaging. When the beams are focused at $\left(y_{f}, z_{f}\right)$, the synthetic time delay function is

$$
\tau(\alpha)=e^{-j k R_{f}}=e^{-j k \sqrt{\left(y_{f}-r \alpha\right)^{2}+\left(z_{f}-r \beta\right)^{2}}} .
$$

Hence, substituting Eqs. (2) and (4) to Eq. (3) defines the synthetic transmit beam pattern of the TRUS-rSAF imaging as follow:

$$
\Psi_{\mathrm{rSAF}}\left(y_{f}, z_{f}\right)=\int_{-\infty}^{\infty} p(\alpha) e^{j k\left(R-R_{f}\right)} d \alpha .
$$

By using the Fresnel approximation, $R-R_{f}$ can be reduced to

$$
R-R_{f}=\frac{y^{2}-y_{f}^{2}}{2 z_{f}}+\frac{r\left(y-y_{f}\right)}{z_{f}} \alpha .
$$

Therefore, substituting Eq. (6) to Eq. (5) yields the final beam profile.

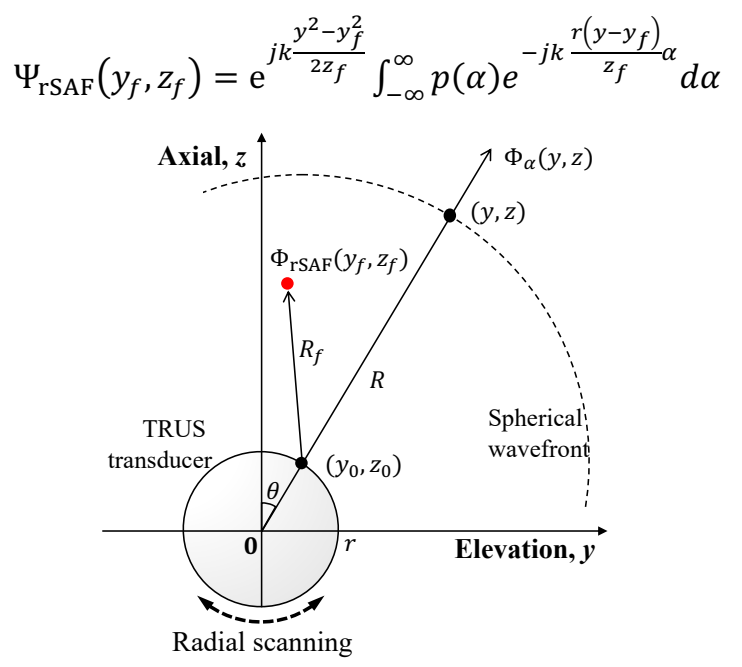

Fig. 1. 2-dimensional theoretical field analysis model of TRUS-rSAF method in elevation-axial plane. Red dot indicates the synthetic focusing pixel $\left(y_{f}, z_{f}\right) ;\left(y_{0}, z_{0}\right)$ is an element position; and $\theta$ is a scanning angle.

$$
=e^{j k \frac{y^{2}-y_{f}^{2}}{2 z_{f}}} \mathcal{F}[p(\alpha)]_{f_{y}=\frac{r y^{\prime}}{\lambda z_{f}}},
$$

where $y^{\prime}=y-y_{f}$. In the equation, the synthetic transmit beam pattern is represented by the Fourier transform $(\mathcal{F}[\cdot])$ of the synthetic angular window function $p(\alpha)$. When assuming $\alpha$ range as $\left[-\frac{1}{2} \alpha_{\max }, \frac{1}{2} \alpha_{\max }\right]$ and uniform element directivity in the radial field without apodization, $p(\alpha)$, can be expressed as

$$
p(\alpha)=\operatorname{rect}\left(\frac{\alpha}{\alpha_{\max }}\right) .
$$

The resultant synthetic transmit beam pattern of the TRUSrSAF imaging is acquired by substituting Eq. (8) to Eq. (7):

$$
\Psi_{\mathrm{rSAF}}\left(y_{f}, z_{f}\right)=\mathrm{c}_{0} \operatorname{sinc}\left(\frac{r \alpha_{\max }}{\lambda z_{f}} y^{\prime}\right),
$$

where $c_{0}=\alpha_{\max } \mathrm{e}^{j k \frac{y^{2}-y_{f}^{2}}{2 z_{f}}}$, and the null-to-null mainlobe width (i.e., $y_{\mathrm{ML}}^{\prime}$ ) is defined by

$$
y_{\mathrm{ML}}^{\prime}=\frac{\lambda z_{f}}{r \alpha_{\max }} .
$$

The equation confirms that the spatial resolution of the TRUSrSAF imaging will be proportional to acoustic wavelength (i.e., the fundamental frequency of TRUS transducer) and imaging depth, while being inversely proportional to rotation radius $r$ and radial synthetic window defined by $\alpha_{\max }$. The grating lobe is not appeared yet as assumed a continuous synthetic angular window $p(\alpha)$.

\section{B. Implementation using virtual sources}

We further practicalized the model into the practical scenario using discrete radial scanning angles, as each angle necessitates an individual transmittance/reception event, rather than being continuous in $\alpha$ domain as in Eq. (8). Here we consider $N$ transmit angles uniformly discretized with an interval $\Delta \alpha$, which can be equated by

$$
p_{s}(\alpha)=\sum_{n=0}^{N-1} \delta\left(\alpha-\alpha_{n}\right),
$$

where $\alpha_{n}=\Delta \alpha \cdot n-\alpha_{\max } / 2$ and $\Delta \alpha=\alpha_{\max } /(N-1)$. Consequently, the synthetic transmit beam pattern of the TRUS-rSAF imaging can be converted to discretized form as

$$
\begin{aligned}
\Psi_{\mathrm{S}}\left(y_{f}, Z_{f}\right) & =e^{j k \frac{y^{2}-y_{f}^{2}}{2 z_{f}}} \sum_{n=0}^{N-1} e^{-j k \frac{r \alpha_{n}}{z_{f}} y} \\
& =c_{0}^{\prime} \frac{\sin \pi \frac{r N \Delta \alpha}{\lambda z_{f}} y}{\sin \pi \frac{r \Delta \alpha}{\lambda z_{f}} y},
\end{aligned}
$$

where $c_{0}^{\prime}=e^{j k \frac{y^{2}-y_{f}^{2}+r \alpha_{\max }}{2 z_{f}} y}$. The null-to-null mainlobe width is given from (12):

$$
y_{s \mathrm{ML}}^{\prime}=\frac{\lambda z_{f}}{r N \Delta \alpha}=\frac{\lambda z_{f}}{r \alpha_{\max }} .
$$

The discretized sampling in $\alpha$ domain results in grating lobes in the beam field positioned at

$$
y_{s \mathrm{GL}}^{\prime}=\frac{\lambda z_{f}}{r \Delta \alpha} n(n=1,2,3, \ldots) .
$$


Therefore, our analytical solution of the TRUS-rSAF imaging unveiled that the most critical yet flexible parameters are $r$ and $\Delta \alpha$, defining theoretical spatial resolution and grating lobe positions. We will perform a heuristic optimization on those critical parameters in the following Section III. Although $\lambda$ also defines the basic spatial resolution and acoustic penetration depth, but we will follow well-established specification in the TRUS-REF imaging.

We here implement the TRUS-rSAF model synthesizing wavefronts diverging from virtual sources (VSs) at a fixed lens focusing point of each radial plane [19]. Fig. 2 defines the revised model of the TRUS-rSAF method. At the VS of each radial plane, the acoustic wavefront forms two-way focusing for transmission and reception, while other depth regions will have spherical wavefront. The wavefront originated from each VS can be synthesized by adjusting time-of-flight passing through corresponding VS $\left(y_{\mathrm{Vs}}, z_{\mathrm{VS}}\right)$ to the target pixel $\left(y_{f}, z_{f}\right)$. Here, the primary consideration for the SAF with 1-D element should be to count only the transmittance planes providing adequate overlap at the target focal point [11], [12]. In synthetic focusing of each radial plane, we define that the distance from the TRUS transducer to VS is $d_{t 1}(i, z)$ at the $i$ th dataset within the synthetic window and $z$ th focusing depth. From the VS, the acoustic wavefront travels to the focusing point $\left(y_{f}, z_{f}\right)$, and we denoted this distance as $d_{t 2}(i, z)$. Therefore, the distance in total transmittance pathway is $d_{t}(i, z)=d_{t 1}(i, z)+d_{t 2}(i, z)$, changing for each synthetic scanning angle, and it yields transmit time-of-flight, $\tau_{t}(i, z)=d_{t}(i, z) / c$, where $c$ is a constant speed of sound in biological tissue (i.e., $1,540 \mathrm{~m} / \mathrm{sec}$ ). On the other hand, an acoustic reception distance $d_{r}$ is defined as the shortest pathway reversing from $\left(y_{f}, z_{f}\right)$ to element in the TRUS transducer $\left(y_{0}, z_{0}\right)$, leading to receiving time-of-flight $\tau_{r}(z)=d_{r}(z) / c$ (i.e., $\left.R_{f}\right)$. In all, adjacent radial planes can be synthesized to the target pixel $\left(y_{f}, z_{f}\right)$ by compensating the focusing delay at each synthetic angle, $\tau_{f}(i, z)=\tau_{t}(i, z)+$ $\tau_{r}(z)$. The coherent synthesis of effective radial planes in a target imaging slice can be equated by

$$
I_{\mathrm{rSAF}}\left(\theta_{n}, z\right)=\frac{1}{N_{\mathrm{syn}}(\mathrm{z})} \sum_{i=n-N_{\text {syn }}(z) / 2+1}^{n+N_{\operatorname{syn}}(\mathrm{z}) / 2} \bar{I}_{i}\left(\theta_{n}, z\right),
$$

where $I_{\mathrm{rSAF}}\left(\theta_{n}, z\right)$ is a signal intensity in a target radial plane at $\theta_{n}$, and $z$ of imaging angle and depth, respectively; $N_{s y n}(z)$ is the number of effective radial planes for the TRUS-rSAF

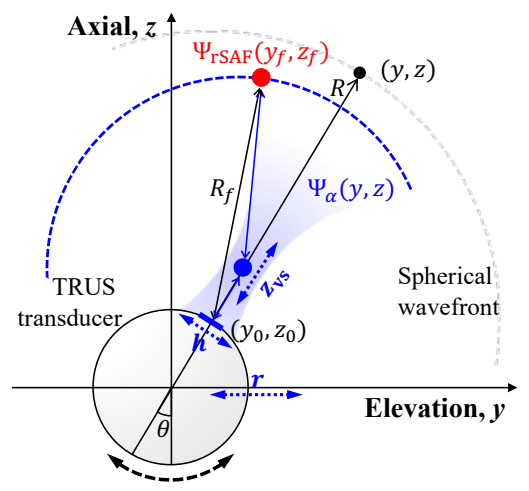

Fig. 2. Optimization of TRUS-rSAF method. Blue indicators show the variables in our rSAF optimization. imaging, defined at specific $z$ by their overlap of adjacent beam profiles; and $\bar{I}_{i}\left(\theta_{n}, z\right)$ is the US intensity at the focal point $\left(\theta_{n}, z\right)$ from $i$ th dataset within the synthetic window which is delay-compensated by $\tau_{f}(i, z)$. The model will be valid for each imaging angle $\theta_{n}(n=1,2, \ldots, N)$, comprising entire imaging volume in an interval of $\Delta \theta$ (i.e., $\Delta \theta=\sin ^{-1} \Delta \alpha$ ).

\section{Simulation AND Optimization}

\section{A. Spatial resolution-oriented optimization strategy}

Eq. (13-1) suggests the critical parameters to define the spatial resolution of the TRUS-rSAF imaging: acoustic wavelength $\lambda$, radial synthetic window defined by $\alpha_{\max }$, and probe radius $r$. In practice, the element height $h$ and $z_{\mathrm{VS}}$ determines $\alpha_{\text {max }}$, and $r$ determines the overlap among adjacent radial planes. Therefore, we performed a heuristic optimization of the individual critical parameter with a specific design objective to maximize the spatial resolution (Fig. 2). Basic design specifications followed those of a clinical TRUS transducer (linear array in BPL9-5/55, BK Ultrasound, Inc., MA, USA): $6.5 \mathrm{MHz}$, center frequency; fractional bandwidth, $80 \% ; 5 \mathrm{~mm}$, elevation aperture size $h ; 20 \mathrm{~mm}$, elevation focusing depth $z_{\mathrm{VS}}$; $10 \mathrm{~mm}$, probe radius $r$. Imaging parameters were as follows: $0.4724^{\circ}$, rotation interval in degree $\Delta \theta ; 128$ or 280 radial scanlines to compose the radial FOVs of $\left[-30^{\circ}, 30^{\circ}\right]$ and $[-$ $66.14^{\circ}, 66.14^{\circ}$ ], respectively. Target imaging depth of interest was from $0 \mathrm{~mm}$ to $70 \mathrm{~mm}$.

During the optimization, clinically relevant ranges for the critical parameters were evaluated to secure its practicality in clinics: $z_{\mathrm{VS}}=\{5,10,15,20,25\} \mathrm{mm} ; h=\{3,4,5,6,7\} \mathrm{mm}$; and $r=\{5,10,15\} \mathrm{mm}$. The optimal $z_{\mathrm{VS}}$ was first defined and then $h$ and $r$ were evaluated independently based on the optimal $z_{\mathrm{Vs}}$. Note that sound propagation speed $c$ was fixed at $1,540 \mathrm{~m} / \mathrm{s}$ during the optimization. In each combinational setup among the parameters, $N_{\text {syn }}(z)$ was determined to secure effective overlap among adjacent beam profiles from multiple transmittance/reception events during radial scanning. Using Field-II simulation of wire targets in a depth range from $10 \mathrm{~mm}$ to $70 \mathrm{~mm}$ depth at $10 \mathrm{~mm}$ interval, we tested each combinational setup while increasing $N_{\text {syn }}$ (i.e., $3,5,7, \ldots$ ) until finding the narrowest radial full-width-half-maximum (FWHM) at a depth of interest [20], [21]. This optimization process was to exclusively synthesize the set of radial planes whose phase information can be effective on a target pixel. A geometrical beam profile model of radial synthetic window was configured using $h, z_{\mathrm{VS}}$, and $N_{\text {syn }}$ derived at the deepest imaging depth, $70 \mathrm{~mm}$ (i.e., $\overline{N_{\text {syn }}}$ ). The model finally defined $N_{\text {syn }}(z)$ giving the corresponding optimal $\alpha_{\max }$ at individual depths $z$ (i.e., $\alpha_{\max }(z)=\Delta \alpha \cdot N_{\text {syn }}(z)$ ). The FWHM at each depth was evaluated.

We also evaluated the impact of the spatial resolutionoriented optimization on signal-to-noise ratio (SNR) in the canonical definition as follows.

$$
\mathrm{SNR}=20 \log _{10} \frac{\mathrm{E}\left[I^{2}\left(\theta_{n}, z\right)\right]}{\sigma^{2}},
$$

where $\mathrm{E}[\cdot]$ is the expectation of the signal amplitude, and $\sigma$ is the noise power. A shallow $z_{\mathrm{VS}}$ may secure high $\alpha_{\max }$ but will 
result in lower acoustic power density in deep tissue than what offered in a clinical TRUS-REF imaging, which deteriorate resultant signal sensitivity. On the other hand, the coherent frame compounding in the TRUS-rSAF framework will bring a counter effect enhancing the signal-to-noise amplitude ratio theoretically with a factor of $\sqrt{N_{\text {syn }}(z)}$ if approximating that $I_{i}\left(\theta_{n}, z\right)=\bar{I}_{i}\left(\theta_{n}, z\right)$ as in Eq. (14).

The SNR at each combinational parameter setup was evaluated in Field-II imaging simulation. For this, a transmit beam profile in each combinational setup was first analyzed to quantify the acoustic energy reaching each depth. We also simulated wire targets located at $10-\mathrm{mm}$ intervals from $10 \mathrm{~mm}$ to $70 \mathrm{~mm}$ to define the signal component. In addition, randomized Gaussian noise image at $-20-\mathrm{dB}$ mean intensity was separately simulated in the corresponding imaging FOV to define the noise component. The SNR deviation in the TRUSrSAF imaging was calculated with a comparison to that in the TRUS-CON imaging where imaging with same specifications but comprising a target volume with only the center radial plane at each scanning angle.

Contrast and information entropy contrast (IEC) were measured in the tissue-mimicking Field-II simulation data for comparative evaluation of TRUS-REF and TRUS-rSAF imaging. Contrast represents deviation between hyperechoic and cyst regions and IEC evaluates spatial acuity quantified by microstructural entropy [22]-[25], which are defined as

$$
\begin{aligned}
& \text { Contrast }=10 \log _{10} \frac{\sum_{y, z \subseteq H R} I^{2}(y, z)}{\sum_{y, z \subseteq C R} I^{2}(y, z)}, \\
& \text { IEC }=\operatorname{InEn} \cdot \hat{C}
\end{aligned}
$$

where HR and CR are hyperechoic mass and anechoic cyst regions, respectively. InEn and $\hat{C}$ are information entropy and mean contrast within the regions-of-interest (ROIs), respectively. InEn and $\hat{C}$ are defined by

$$
\begin{gathered}
\operatorname{InEn}=-\sum_{i=\hat{I} \min }^{\hat{I}_{\max }} \operatorname{Prob}\left(\hat{I}_{i}\right) \cdot \log _{2}\left[\operatorname{Prob}\left(\hat{I}_{i}\right)\right], \\
\hat{C}=\frac{1}{\left(K_{y}-1\right)\left(K_{z}-1\right)} \sum_{y=0}^{K_{y}-2} \sum_{z=0}^{K_{z}-2}|p(y, z)-p(y, z+1)|,
\end{gathered}
$$

where, $\hat{I}_{\min }, \hat{I}_{\max }$ are the minimum and maximum gray scale pixel intensities, $\operatorname{Prob}\left(\hat{I}_{i}\right)$ is the probability of pixel distribution at $i$ th gray level, and $p(y, z)$ is the pixel intensity at $(y, z)$ coordinate.

\section{B. Critical parameter optimization \\ 1) Impact of $z_{V S}$}

The transmit beam profiles of the TRUS-rSAF imaging were first analyzed as a function of $z_{\mathrm{VS}}$. 1-D radial intensity profiles were extracted at $\{30,50,70\} \mathrm{mm}$ using the Field-II simulation of transmit beam pattern (Fig. 3(a)). The transmit beam profiles become weaker as $z_{\mathrm{VS}}$ gets shallower, sacrificing the signal sensitivity at deep depth. For example, $z_{\mathrm{VS}}=\{5,10,15,20\}$ $\mathrm{mm}$ provided $\{92.75,79.68,47.24,13.50\} \%$ and $\{90.25$, $76.40,62.84,29.69\} \%$ of reductions of maximal acoustic power reaching to at 30- and 70-mm depths, respectively, when compared to the case with $z_{\mathrm{Vs}}$ at $25 \mathrm{~mm}$ (Fig. 3(b)). This will affect the signal component for SNR calculation. On the other hand, a transmit beam profile had wider beam distribution when shallower $z_{\text {VS }}$ was applied, which resulted in a greater $\overline{N_{\text {syn }}}$ : $\{49,23,11,7,5\}$ (i.e., $\theta_{\max }=\left\{22.67^{\circ}, 10.39^{\circ}, 4.72^{\circ}, 2.83^{\circ}\right.$, $\left.1.89^{\circ}\right\}$ ) when $Z_{\mathrm{VS}}=\{5,10,15,20,25\} \mathrm{mm}$, respectively. Therefore, a net SNR will be determined by a competing effect of transmit power and synthetic window width specified by $z_{\mathrm{VS}}$, as shown in Fig. 3(c).

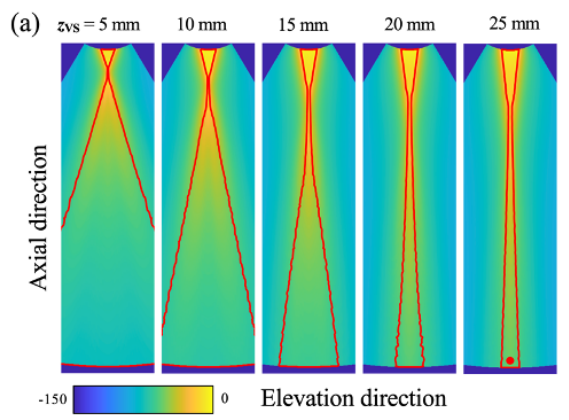

(d)

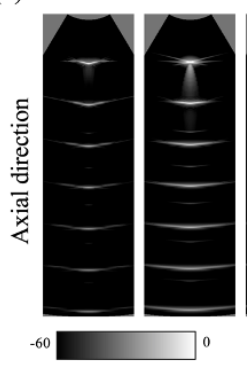

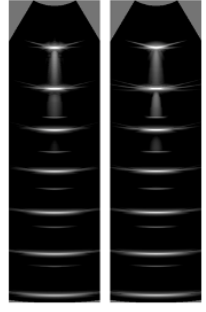

Elevation direction (b)

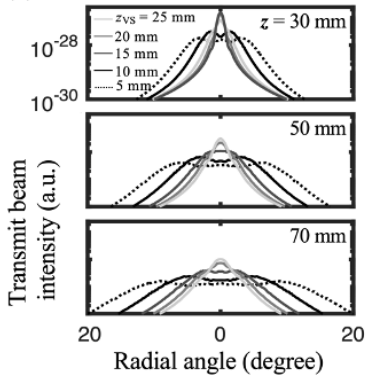

(e)
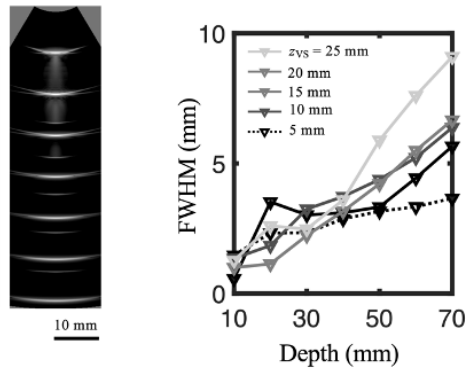

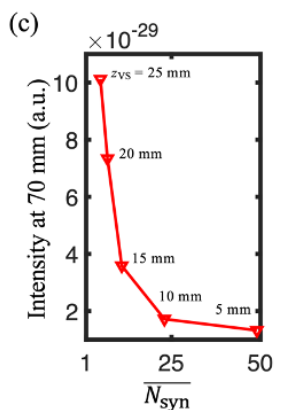

(f)

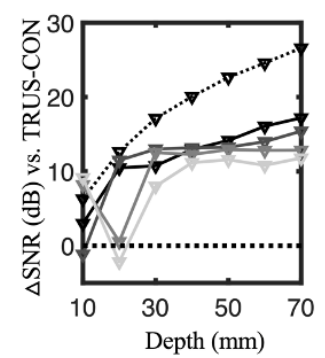

Fig. 3. Critical parameter optimization on $\boldsymbol{z}_{\mathrm{VS}}$ impact on TRUS-rSAF imaging performance. (a) Transmit beam profiles at different $z_{\mathrm{VS}}$. Red lines indicate -6-dB contour from each depth. Intensity was normalized to the maximum when $z_{\mathrm{Vs}}=25 \mathrm{~mm}$. (b) Transmit beam intensity at 30-, 50-, and 70-mm depths. (c) Beam intensity as a function of $\overline{N_{\text {syn }}}$ at different $z_{\mathrm{Vs}}$ on the red observation point in (a). (d) Field-II wire-target simulation data at different $z_{\mathrm{Vs}}$. (e) Full-widthhalf-maximum (FWHM) over imaging depth. (f) SNR difference between the TRUS-rSAF and TRUS-CON imaging over imaging depth. 
Fig. 3(d) shows the 2-D TRUS-rSAF images reconstructed in elevation-axial direction. In the visual assessment, the TRUSrSAF imaging showed more substantial enhancement when shallower $z_{\mathrm{VS}}$ was applied. The FWHM measurement at each depth quantitatively validated the trend (Fig. 3(e)). For example, at 70-mm depth, the FWHM of the target was $\{3.66$, $5.66,6.37,6.66,9.10\} \mathrm{mm}$ at $Z_{\mathrm{VS}}=\{5,10,15,20,25\} \mathrm{mm}$, respectively. In general observation, the TRUS-rSAF provides additional spatial acuity in the post-focal depth range by extending the synthetic transmit aperture.

It should be noted that the SNR definition in Eq. (15) requires the equal attention to noise components, which should be related to the coherent compounding effect with $N_{\text {syn }}(z)$. Fig. 3(f) shows the SNR difference between the TRUS-CON and TRUS-rSAF imaging with different $z_{\mathrm{VS}}$. It was clearly notable that more significant SNR improvements were obtained in the TRUS-rSAF imaging at deeper imaging depth with shallower $Z_{\mathrm{VS}}$. For example, $\{6.28,17.13,22.66,26.60\}$-dB higher SNRs were achieved at $\{10,30,50,70\}-\mathrm{mm}$ depths when $z_{\mathrm{VS}}=5$ $\mathrm{mm}$, whereas there were only $\{9.14,8.01,11.58,11.80\}-\mathrm{dB}$ improvements when $z_{\mathrm{VS}}=25 \mathrm{~mm}$.

Note that there was no further analysis on grating lobe with $Z_{\mathrm{VS}}$ as no impact is expected given with Eq. (13-2).

\section{2) Impact of $h$}

Fig. 4(a) shows the transmit beam profiles at different $h=\{3$, $4,5,6,7\} \mathrm{mm}$. Note that the $z_{\mathrm{VS}}$ is fixed at $5 \mathrm{~mm}$ based on the previous optimization, and other parameters were used as given in the clinical reference TRUS transducer. The acoustic power when $h=\{4,5,6,7\} \mathrm{mm}$ was reduced by $\{12.64,21.51,24.24$, $30.74\} \%,\{6.95,14.65,16.21,23.37\} \%$, and $\{0.61,3.03,12.73$ $16.97\} \%$ at 30-, 50-, and 70-mm depths, respectively, when compared to the corresponding cases of having $h=3 \mathrm{~mm}$ (Fig. 4(b)). Notably, the intensity changes among $h=\{4,5,6,7\} \mathrm{mm}$ were not simply inversely proportional given by the dependency to the different transmit aperture sizes (Fig. 4(c)). Therefore, a net SNR at each depth will be determined by a combination of the transmit power and $N_{\text {syn }}(z)$.

The spatial resolution was evaluated for the corresponding setups using the Field-II simulation data of wire targets (Fig. $4(\mathrm{~d}))$. The $\overline{N_{\text {syn }}}$ was proportional to $h:\{23,37,51,61,65\}$ for $h=\{3,4,5,6,7\} \mathrm{mm}$, which was predictable with the divergence of focused wavefront proportional to the transmit aperture width (Fig. 4(b)). The FWHMs measured at each depth indicated the inversely proportional relationship between FWHM and $h$ (Fig. 4(e)). For example, the FWHMs of the target at $30 \mathrm{~mm}$ are $\{2.92,2.50,2.17,1.68,1.48\} \mathrm{mm}$ for $h=$ $\{3,4,5,6,7\} \mathrm{mm}$, respectively. At 70-mm depth, the FWHMs became $\{7.76,4.61,3.77,3.66,3.92\} \mathrm{mm}$ for $h=\{3,4,5,6,7\}$ $\mathrm{mm}$, respectively. Therefore, the larger $h$ will provide more consistent and higher spatial resolution.

Fig. 4(f) shows the SNR of the TRUS-CON and TRUS-rSAF imaging with different $h$. The higher SNRs were obtained when applied the TRUS-rSAF imaging with wider $h$. Representatively, $\{5.104,16.12,21.59,25.26\}$-dB higher SNRs were achieved at $\{10,30,50,70\}-\mathrm{mm}$ depths when $h=7 \mathrm{~mm}$, whereas there were only $\{10.73,12.84,16.95,18.95\}-\mathrm{dB}$ improvements when $h=3 \mathrm{~mm}$. Therefore, the change in $h$ affects SNR combinatorially by acoustic divergency and total acoustic power transmittance.

Note that there was no further analysis on the grating lobe with $h$ as no impact is expected with Eq. (13-2).

\section{3) Impact of $r$}

Our analytical solution unveiled that $r$ is the most critical parameter affecting spatial resolution and grating lobe positions. We performed a heuristic optimization by testing a range of $r$ at $\{5,10,15\} \mathrm{mm}$ (Fig. 5(a)). As predicted in Eq. (13), the measured FWHMs of the wire target at each depth presented the inversely proportional relationship between

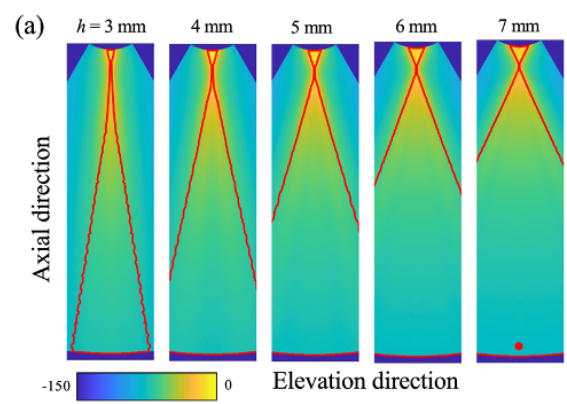

(d)

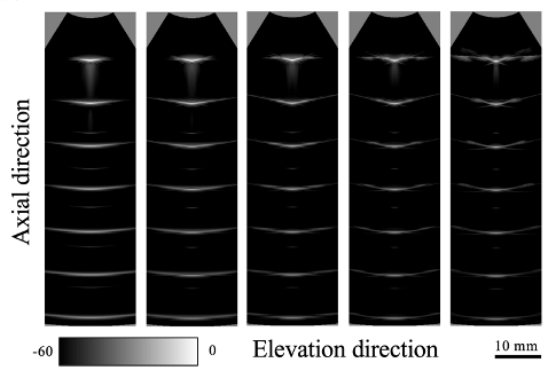

(b)
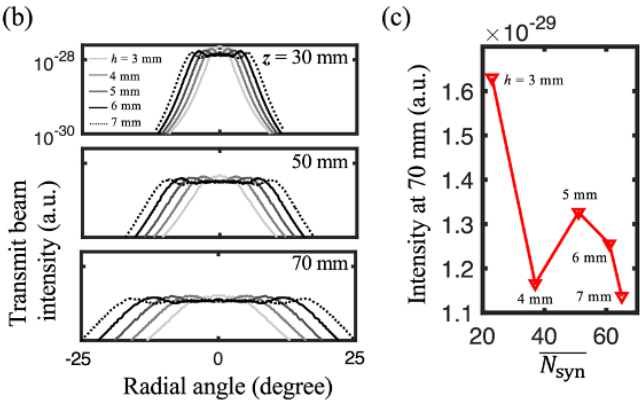

(e)

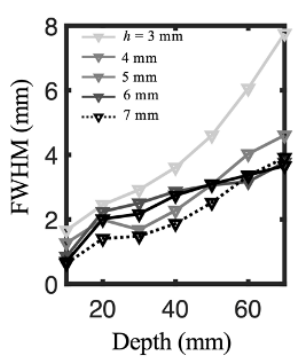

(c)

(f)

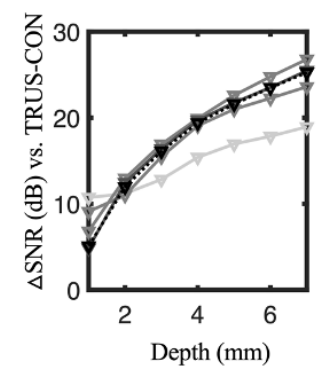

Fig. 4. Critical parameter optimization on $\boldsymbol{h}$ impact on TRUS-rSAF imaging performance. (a) Transmit beam profiles at different $h$. Red lines indicate -6$\mathrm{dB}$ contour from each depth. Intensity was normalized to the maximum when $h=3 \mathrm{~mm}$. (b) Transmit beam intensity at 30-, 50-, and 70-mm depths. (c) Beam intensity as a function of $\overline{N_{\text {syn }}}$ at different $h$ on the red observation point in (a). (d) Field-II wire-target simulation data at different $h$. (e) Full-width-half-maximum (FWHM) over imaging depth. (f) SNR difference between the TRUS-rSAF and TRUS-CON imaging over imaging depth. 
(a)

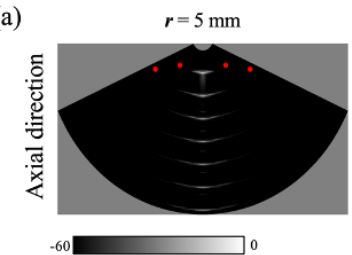

(b)

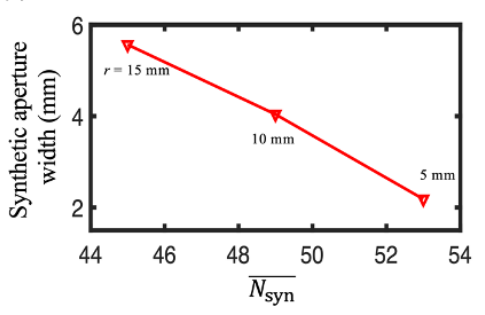

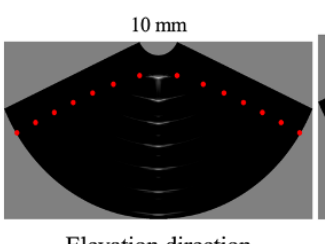

Elevation direction

(c)

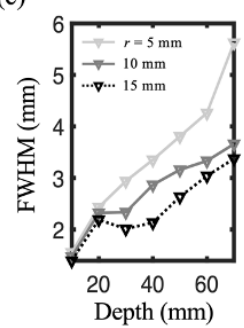

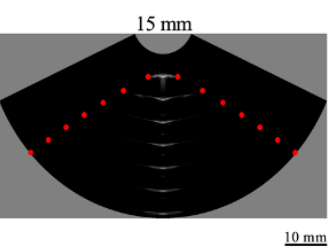

(d)

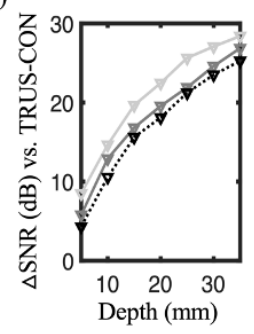

(e)

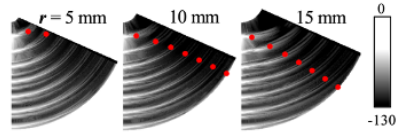

(f)
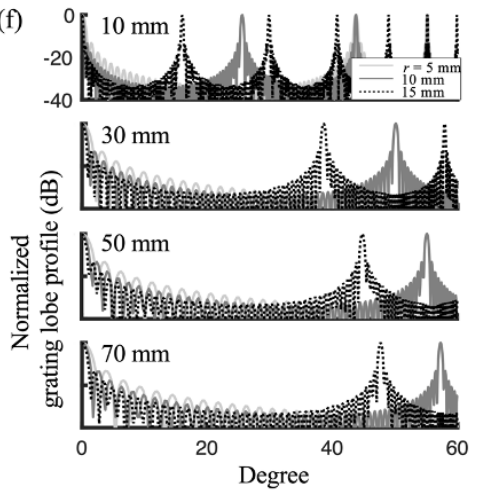

Fig. 5. Critical parameter optimization on $r$ impact on TRUS-rSAF imaging performance. (a) Field-II wire-target simulation data at different $r$. (b) Aperture size as a function of given $\overline{N_{\text {syn }}}$. (c) Full-width-half-maximum (FWHM) over imaging depth. (d) SNR difference between the TRUS-rSAF and TRUS-CON imaging over imaging depth. (e) TRUS-rSAF images with extended dynamic range. Red dots indicate the first grating lobe positions. (e) Theoretical grating lobe positions at 10-, 30-, 50-, and 70-mm depths and at different $r$.

FWHM and $r$ as shown in Fig. 5(c). FWHMs at 30-, 50-, and $70-\mathrm{mm}$ depths indicated $\{2.94,2.34,2.01\} \mathrm{mm},\{3.81,3.16$, $2.64\} \mathrm{mm}$, and $\{5.63,3.66,3.38\} \mathrm{mm}$ when $r$ is $\{5,10,15\}$ $\mathrm{mm}$, respectively. It can be explained by an interesting observation that the $\overline{N_{\text {syn }}}$ is inversely proportional to $r$ when fixed $\Delta \theta$ at $0.4724^{\circ}:\{5349,45\}$ for $r=\{5,10,15\} \mathrm{mm}$, leading to the corresponding radial aperture sizes at $\{2.18,4.04,5.57\}$ mm, respectively (Fig. 5(b)).

SNR was also calculated at each $r$. Note that $r$ will not affect the individual radial plane, so that the SNR improvements should be sorely determined by $N_{\text {syn }}(z)$. Fig. 5(d) present the SNR proportional to $\overline{N_{\text {syn }}}$. For example, 28.39-, 26.87-, 26.26$\mathrm{dB}$ SNR improvements were obtained at 70-mm depth when $r$ at $\{5,10,15\} \mathrm{mm}$, respectively.

We consider another crucial impact of $r$ on the positions of grating lobes. Our analytic solution in Eq. (13-2) suggests that the enlarged $r$ shifts the grating lobes toward the mainlobe. Bmode images with extended dynamic range (Fig. 5(e)) validates the theoretical expectation matched with theoretical synthetic transmit beam patterns in Fig. 5(f). The first grating lobe positions when $r=\{5,10,15\} \mathrm{mm}$ were at $\pm\{43.78,25.63$, $15.99\}^{\circ}, \pm\{64.03,50.11,38.62\}^{\circ}, \pm\{66.97,55.19,44.88\}^{\circ}$, and $\pm\{68.10,57.30,47.67\}^{\circ}$ at $\{10,30,50,70\}-\mathrm{mm}$ depths, respectively. Therefore, the TRUS-rSAF imaging with a larger $r$ results in more substantial grating lobe artifacts.

\section{PRACtical Implementation}

In previous sections, we established the analytical approach to unveil and optimize the critical parameters: $z_{\mathrm{VS}}=5 \mathrm{~mm}, h=$ $7 \mathrm{~mm}$, and $r=15 \mathrm{~mm}$. In this section, we present an integrative optimization and effectuation of the TRUS-rSAF imaging with a specific comparison to the TRUS-REF imaging that illustrates a basic expectation in clinics.

The TRUS-rSAF imaging provided the optimal $\overline{N_{\text {syn }}}$ at 65 , and resulted in the $\{46.67,-11.15,15.65,46.75,54.85,52.79$, $51.00\}-\%$ narrower FWHMs at $\{10,20,30,40,50,60,70\}-\mathrm{mm}$ depths compared to those by the TRUS-REF imaging, respectively (Fig. 6(e)). To validate clinical efficacy of the volumetric TRUS-rSAF imaging, we compared the resultant radial imaging resolution at each depth with that by the TRUS imaging using an in-plane microconvex array (BPC8-4/10) in the bi-planar TRUS transducer equipped with the linear array for our TRUS-rSAF imaging (BPL9-5/55): number of channel, $128 ; 6 \mathrm{MHz}$, center frequency; fractional bandwidth, $60 \%$; element pitch, $0.21 \mathrm{~mm}$; element height, $7 \mathrm{~mm}$; radius of curvature, $10 \mathrm{~mm}$; f-number in transmit aperture, 3 . Encouragingly, the TRUS-rSAF imaging provided the spatial resolution even higher than the in-plane microconvex TRUS imaging: $\{53.26,-1.08,4.92,23.93,34.64,29.31,24.78\}-\%$ narrower FWHMs at $\{10,20,30,40,50,60,70\}$-mm depths. However, given the current imaging configuration (i.e., $\Delta \theta=$ $0.4724^{\circ}$ ), the TRUS-rSAF image suffers from grating artifacts due to coarse radial sampling. In addition, low SNR at deep imaging depths, primarily due to diverging beam field designed to secure wider synthetic window, should also be addressed.

In the following subsections, we will present further optimization to control the grating lobe positions and $N_{\text {syn }}(z)$, while preserving $\alpha_{\max }$ to secure the highest spatial resolution. The corresponding change in volume scanning rate will be also analyzed.

\section{A. Grating lobe}

Fig. 6(a) shows the grating lobe positions in the optimized TRUS-rSAF imaging at $\Delta \theta /\{1.00,1.25,1.50,1.75,2.00$, $3.02\}$ (i.e., $\left\{0.4724^{\circ}, 0.3779^{\circ}, 0.3149^{\circ}, 0.2699^{\circ}, 0.2362^{\circ}\right.$, $\left.\left.0.1564^{\circ}\right\}\right)$. We identified that the original $\Delta \theta$ at $0.4724^{\circ}$ presented substantial grating lobe artifacts, but its positions shifted far from the target as the finer $\Delta \theta$ is applied. Red dots indicate the theoretical grating lobe positions at each target depth, and they were well matched with the radial profiles of the wire target signal (Fig. 6(c)). Therefore, our analytical approach can provide a theoretical foundation to design an effective $\Delta \theta$ control to alleviate the grating lobe artifacts.

We herein set our performance benchmark by designing the first grating lobe positions out of $-20 \mathrm{~dB}$ radial beam profile of 
(a)

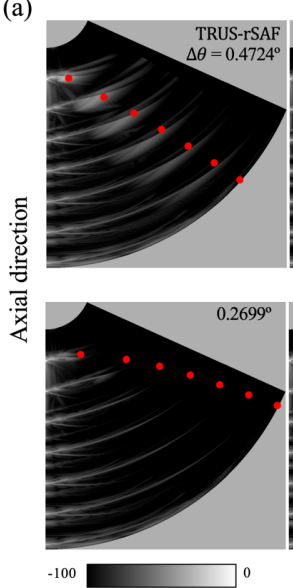

(d)
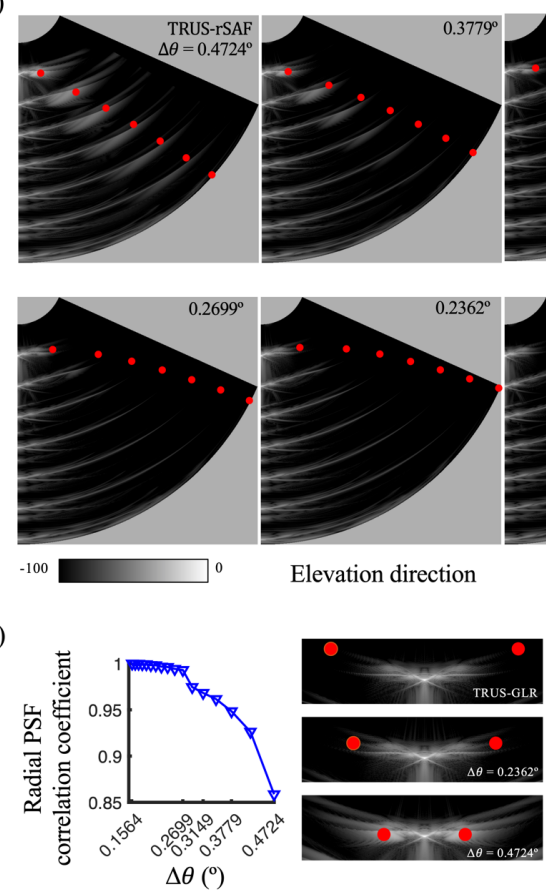

Elevation direction (b)
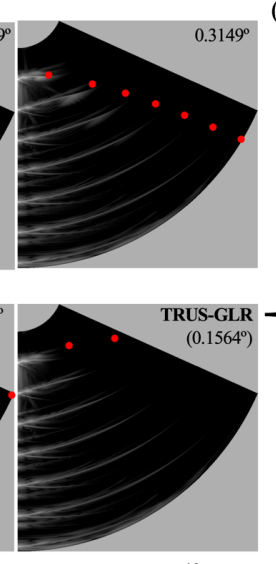

(e)

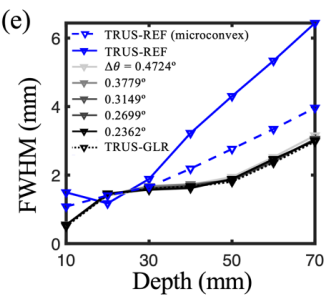

(b)
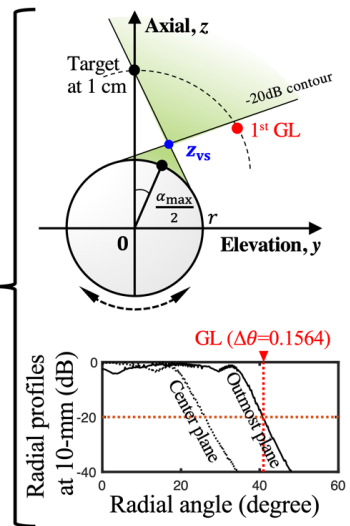

(c)

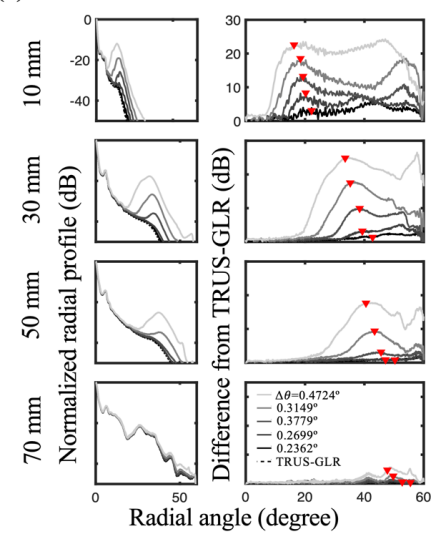

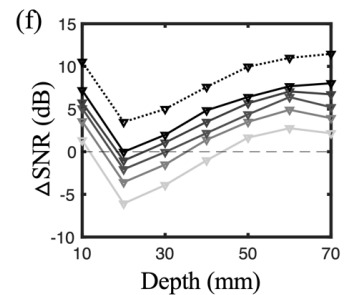

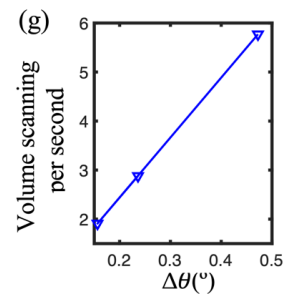

Fig. 6. Global optimization of the TRUS-rSAF imaging performance. (a) Field-II wire-target simulation data at different $\Delta \theta$ (FOV $\left.=\left[0^{\circ}, 66.14^{\circ}\right]\right)$. Red dots indicate the first grating lobe positions; (b) Definition of grating-lobe-rejected TRUS (TRUS-GLR) imaging having the first grating lobe position out of outmost plane; (c) (Left) Radial profiles at different depth. (Right) Corresponding grating lobe intensity profiles subtracted by TRUS-GLR. Red marks indicate the first grating lobe positions (d) 2-D cross-correlation between the PSF of TRUS-GLR and others at 10mm. (e) Full-width-half-maximum (FWHM) over imaging depth. (f) SNR difference between TRUS-rSAF and TRUS-REF over imaging depth. (g) Estimated volume rate at different $\Delta \theta$. (h) Field-II wire-target simulation data of TRUS-REF, TRUS-rSAF, and TRUS-GLR.

the outmost radial plane in the synthetic window, by which the grating lobe artifacts can be effectively rejected (TRUS-GLR, Fig. 6(b)). We set a strict design criterion to reject the grating lobe artifacts at $z_{f}=10 \mathrm{~mm}$. Grating lobes at imaging depths deeper than the target depth will be also suppressed, following the proportional relationship of the grating lobe positions to imaging depth $z_{f}$ in Eq. (13-2). Fig. 6(b) shows the single-sided radial beam profiles of the center plane and the outmost plane. The condition defines the corresponding $\Delta \theta$ at $0.1564^{\circ}$ producing the grating lobe positions at $40.96^{\circ}$. Corresponding B-mode image (i.e., TRUS-GLR) presented a successful rejection of grating lobe artifacts, when compared to the original design with $\Delta \theta$ at $0.4724^{\circ}$ (Fig. 6(a)). In addition, 2-D cross-correlation coefficients measured between point-spread functions of TRUS-GLR and other TRUS-rSAF images at 10$\mathrm{mm}$ depth presented noticable drop from $\Delta \theta=0.2699^{\circ}$, which implies the significant grating lobe artifact (Fig. 6(d)).

\section{B. Signal-to-noise ratio}

As discussed in the previous sections, the SNR of the TRUS$\mathrm{rSAF}$ imaging is lowered by the diverging acoustic transmission but can be compensated by increasing $N_{\text {syn }}(z)$. Fig. 6(f) presents the SNR difference between TRUS-rSAF and -REF imaging from the wire targets over imaging depths. Note that the change of $\Delta \theta$ did not affect the spatial resolution by fixing $\alpha_{\text {max }}$ (i.e., $\theta_{\max }=30.23^{\circ}$ with $\overline{N_{\text {syn }}}:\{65,81,97,113$, $129,195\}$ at $\Delta \theta:\left\{0.4724^{\circ}, 0.3779^{\circ}, 0.3149^{\circ}, 0.2699^{\circ}, 0.2362^{\circ}\right.$, $\left.0.1564^{\circ}\right\}$, Fig. 6(e)).
Here we tested our design criterion to provide SNR comparable to that in the TRUS-REF imaging, by which the basic expectation in modern clinical urology can be met. If we aim to have comparable or higher SNR at entire depth range, the minimal $\Delta \theta$ should be $0.2362^{\circ}$. On the other hand, one may focus on deep tissue at $60 \mathrm{~mm}$, given that the superficial region will have less acoustic attenuation. However, the condition is already achieved when $\Delta \theta=0.4724^{\circ}$ in TRUS-rSAF imaging.

\section{Volume scanning rate}

Although the strategy reducing $\Delta \theta$ was effective both to alleviate grating lobes and to enhance SNR, it presents a drawback in volume scanning rate. To illustrate, we first estimated a conventional scanning time with the traditional imaging specifications: 8-cm imaging depth; 5-plane-wave angle compounding in lateral dimension; 280 scanlines at $\Delta \theta$ of $0.4724^{\circ}$, constructing FOV over $\left[-66.14^{\circ}, 66.14^{\circ}\right] ; \theta_{\max }$ of $30.23^{\circ}$ (i.e., $\overline{N_{\text {syn }}}$ at 65 ). Note that mechanical and electrical transition time is not considered here for simpler presentation. The estimation starts from the round-trip time duration for the imaging depth (i.e., $6.49 \mu \mathrm{sec} / \mathrm{cm}$ acoustic propagation speed $\times$ $8 \mathrm{~cm} \times 2=103.90 \mu \mathrm{sec})$ plus a redundant duration between radial planes (assumed to be $20 \mu \mathrm{sec}$ ), multiplied by the number of lateral compounding events $(123.90 \mu \mathrm{sec} \times 5$ compounding events $=619.50 \mu \mathrm{sec})$, leading to volume scanning time with the number of radial planes composing the volume (i.e., 619.50 $\mu \mathrm{sec} \times 280$ planes $=173.46 \mathrm{msec})$. In this case, a volume scanning rate is 5.77 volume/second.

Here we include the further optimization on grating lobe rejection. TRUS-GLR imaging suggests $\Delta \theta$ at $0.1564^{\circ}$ for the 
(a)

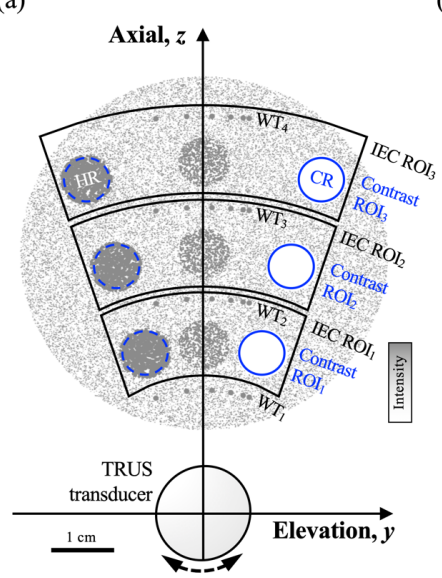

(b)

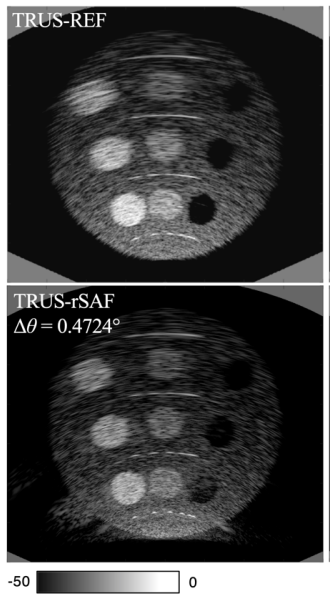

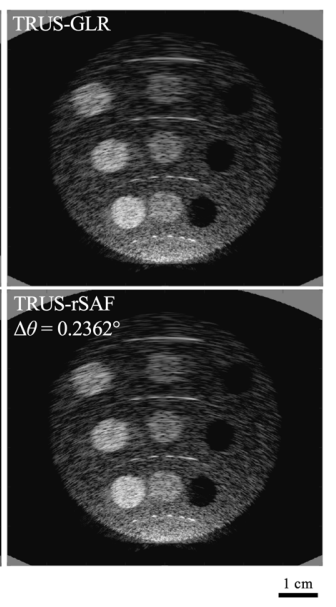

(c)

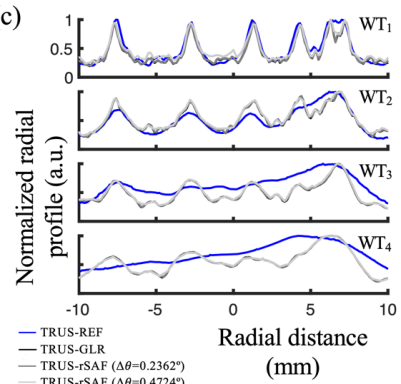

(d)

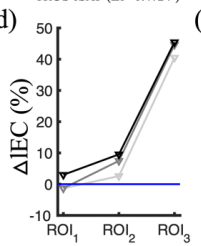

(e)

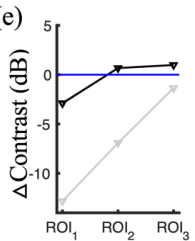

Fig. 7. Evaluation of TRUS imaging simulation. (a) Geometrical illustration of the prostate-mimicking phantom. HR: Hyperechoic region. CR: Cyst region. WT: Wire target. (b) TRUS images of simulated prostate imaging scenario. White dashed-line boxes represent the ROIs centered at 20-, 35-, and 50- mm, for measuring IEC (c) Normalized 1-D profiles of $\mathrm{WT}_{1}(12.5 \mathrm{~mm}), \mathrm{WT}_{2}(27.5 \mathrm{~mm}), \mathrm{WT}_{3}(42.5 \mathrm{~mm})$, and $\mathrm{WT}_{4}(57.5 \mathrm{~mm})$.

effective rejection starting from $10 \mathrm{~mm}$. In this case, the volume scanning times will be $524.10 \mathrm{msec}$, which is $202.14 \%$ longer than that by the original specifications (i.e., $173.46 \mathrm{msec}$ ). The corresponding volume scanning rate is 1.91 volume/second.

On the other hand, our SNR optimization of TRUS-rSAF required the minimal $\Delta \theta$ at $0.2362^{\circ}$ (Fig. 6(f)). In this case, the volume scanning time will be $346.92 \mathrm{msec}$, leading to the $50 \%$ reduction in the imaging rate (i.e., 2.88 volume/second).

Given the tradeoff among multiple metrics (i.e., SNR, grating lobe, volume scanning rate), users of the TRUS-rSAF imaging must prioritize their imaging specifications when determining $\Delta \theta$ in the TRUS-rSAF imaging.

\section{Tissue-mimicking simulation}

A prostate tissue-mimicking simulation was performed in Field-II simulation to evaluate practical performance of TRUSrSAF framework. Fig. 7(a) shows the ground-truth field definition with wire targets (WT), hyperechoic mass (HR), and hypoechoic cyst (CR). Fig. 7(b) demonstrates the simulated Bmode images of TRUS-REF and TRUS-rSAF with different scanline intervals: $\Delta \theta=0.4724^{\circ}, 0.2362^{\circ}$ and $0.1564^{\circ}$ (TRUSGLR). In visual assessment, apparent enhancement in spatial resolution was identified in the TRUS-GLR imaging. Relative improvements over the TRUS-REF imaging were more obvious in the deeper imaging depth. Fig. 7(c) shows the 1-D radial profiles of the wire targets $\left(\mathrm{WT}_{1-4}\right)$, agreeing with the expectation from Fig. 6(e) - The TRUS-rSAF well resolved $<$ 3-mm distance in entire imaging depth regardless of $\Delta \theta$. Otherwise, the TRUS-REF imaging even failed to resolve 5$\mathrm{mm}$ distance between targets from 50-mm depth. On the other hand, a significant amount of grating lobe artifacts was found in the TRUS-rSAF when $\Delta \theta=0.4724^{\circ}$ as expected. The TRUSrSAF with the optimized $\Delta \theta$ at $0.2362^{\circ}$ showed the image quality comparable to that in the TRUS-GLR imaging with effective suppression of the grating lobe artifacts.

Quantitative evaluations were performed to validate the visual observations. Contrast between the hyperechoic and hypoechoic targets in Fig. 7(e) indicated $\{30.08,27.24,25.12\}$ $\mathrm{dB}$ in the TRUS-REF imaging at $\mathrm{ROI}_{1-3}$. On the other hand, the
TRUS-rSAF imaging showed $\{17.27,20.32,23.78\} \mathrm{dB},\{27.14$, $27.92,26.09\} \mathrm{dB}$, and $\{27.20,27.92,26.11\} \mathrm{dB}$ at $\left\{\mathrm{ROI}_{1}, \mathrm{ROI}_{2}\right.$, $\left.\mathrm{ROI}_{3}\right\}$ when $\Delta \theta=0.4724^{\circ}, 0.2362^{\circ}$, and $0.1564^{\circ}$, respectively. Notably, the TRUS-rSAF imaging at $\Delta \theta=0.4724^{\circ}$ presented low Contrast value due to severe grating lobe artifacts. Otherwise, the TRUS-GLR $\left(\Delta \theta=0.1564^{\circ}\right)$ and the optimized TRUS-rSAF imaging $\left(\Delta \theta=0.2362^{\circ}\right)$ successfully suppressed the grating lobe artifacts, resulted in the higher Contrast values than the TRUS-REF imaging in $\mathrm{ROI}_{2-3}$. The TRUS-REF imaging provided the higher Contrast value at $\mathrm{ROI}_{1}$ due to the lower side lobe artifacts by tightly-focused single transmit/receive event, but the lower spatial resolution at other imaging depths should be reminded (Fig. 6(e) and Fig. 7(c)) .

The IEC measurements at different depth range in Fig. 7(d) indicated the significant increase of visual information due to the TRUS-rSAF imaging. The IEC ROIs centered at $\{20,35$, $50\}$-mm depths (i.e., $\mathrm{ROI}_{1}, \mathrm{ROI}_{2}$, and $\mathrm{ROI}_{3}$ ) were $\{2.21,0.74$, $0.25\} \mathrm{e}^{-2}$ for TRUS-REF; $\{2.18,0.76,0.36\} \mathrm{e}^{-2},\{2.18,0.80$, $0.37\} \mathrm{e}^{-2}$, and $\{2.28,0.81,0.37\} \mathrm{e}^{-2}$ for TRUS-rSAF when $\Delta \theta=$ $0.4724^{\circ}, 0.2362^{\circ}$ and $0.1564^{\circ}$. The corresponding fractional improvements over the TRUS-REF imaging were $\{-1.32,-1.31$, $2.99\} \%$ in $\mathrm{ROI}_{1},\{2.59,7.47,9.54\} \%$ in $\mathrm{ROI}_{2}$, and $\{40.48$, $44.62,45.43\} \%$ in $\mathrm{ROI}_{3}$ for $\Delta \theta=\left\{0.4724^{\circ}, 0.2362^{\circ}, 0.1564^{\circ}\right\}$, respectively. The results indicate that the TRUS-rSAF and TRUS-REF imaging delivers comparable amount of visual information at $20 \mathrm{~mm}$ depth, but the TRUS-rSAF clearly outperform in the deeper imaging depth ranges.

\section{Discussions and Conclusion}

In this paper, we demonstrated an analytical approach to optimize the volumetric TRUS imaging using radial synthetic aperture focusing technique. The closed-form analytical model identified the critical parameters (i.e., $z_{\mathrm{VS}}, h$, and $r$ ), affecting the TRUS-rSAF imaging spatial resolution and grating lobe positions (Eqs. (13-1) and (13-2)). Heuristic optimization of the individual critical parameters well agreed with the model (Figs. $3,4,5)$. The quantitative perspectives obtained from the analytical approach led to the significantly higher spatial resolution than what given with the TRUS-REF imaging, and 
even outperformed the in-plane microconvex TRUS imaging (Fig. 6). The impacts of such spatial resolution-oriented optimization on the SNR and grating lobe artifacts were also analyzed and optimized. From the analytical model, we identified that $\Delta \theta$ took an important role to define the SNR and the first grating lobe positions. Further specific TRUS-rSAF imaging strategy was established to recover the SNR to the level by the TRUS-REF imaging and also to remove the grating lobe artifacts in depths of interest by controlling the radial scanning density. Moreover, a prostate mimicking phantom was simulated to evaluate its clinical effectiveness of the optimized TRUS-rSAF. In addition to the higher spatial resolution compared to TRUS-REF, TRUS-rSAF provided better image interpretation performance. In specific, TRUS-rSAF with reduced $\Delta \theta$ provided higher contrast at deeper imaging depth and IEC value giving more visual information.

We plan several further works to elaborate the TRUS-rSAF imaging framework for tangible impact in clinics. (1) A customized TRUS transducer with the optimized parameters (i.e., $r=15 \mathrm{~mm} ; h=7 \mathrm{~mm} ; z_{\mathrm{Vs}}=5 \mathrm{~mm}$ ) will be first implemented. An effective radial scanning configuration will be embedded to provide the target $\Delta \theta$ for sufficient SNR, grating lobe suppression, and volume scanning rate. The developed system will be evaluated in practical circumstances imaging large animal models and/or human subjects. (2) We will also identify the efficacy of the analytical model to describe the resource-limited volumetric imaging circumstance. For example, the radial scanning accuracy to locate individual planes is dependent to the performance of a rotational actuator, and the framework necessitate its sophisticated integration to a TRUS transducer, which will increase the overall cost. We will develop an efficient TRUS imaging system that can provide a robust imaging performance using a low-cost actuator or an external angle tracker. The analytical model will be evolved to reflect the uncertainty of the low-accuracy angle tracking. In detail, the scanning errors of various tracking solutions will be stochastically modelled to be adjusted in Eq. (2). The mathematical derivation will produce the resultant shift of the synthesized beam profile modified from Eq. (7). This analytical approach will again result in a practical strategy to alleviate the volumetric imaging accuracy. The successful development of the low-cost TRUS imaging will produce a synergy with an integration to our ultra-compact US imaging solutions [26][28].

We will further generalize our analytical description of the radial scanning configuration towards enabling a synthetic tracked aperture ultrasound (STRATUS) imaging framework, in which volumetric synthetic window is arbitrarily changing by a tracked robotic arm [29]. The analytical model of the TRUS-rSAF imaging may take an essential part to describe the radial synthesis of arbitrary planes. Such comprehensive analytical approach in describing and optimizing an arbitrary SAF in translational and rotational dimensions will open a new opportunity to develop more reliable robot-assisted US imaging diagnostics.

We also envision the application of the developed TRUSrSAF method beyond conventional US diagnostics. Photoacoustic (PA) imaging is emerging modality that can provide rich optical contrast at high spatiotemporal resolution in deep biological tissue [30], [31]. Thanks to its hybrid mechanism between optics and acoustics, novel clinical and scientific applications have been investigated extensively [32][35]. Our group recently presented a molecular PA imaging of prostate-specific membrane antigen (PSMA) which is overexpressed in aggressive prostate tumor [36], [37]. We will further merge the analytical approach to optimize TRUS-rSAF imaging for localizing PSMA expression at high spatiotemporal and contrast resolution, which will secure higher clinical sensitivity and specificity.

In all, we concluded that the proposed TRUS-rSAF imaging framework will provide a breakthrough to provide the highdefinite and high-contrast urological diagnostics in clinics. Also, the analytical approach will open enormous opportunities to expand its efficacy towards a low-cost TRUS imaging solution or molecular imaging of prostate cancer.

\section{REFERENCES}

[1] E. J. Trabulsi, D. Sackett, L. G. Gomella, and E. J. Halpern, "Enhanced Transrectal Ultrasound Modalities in the Diagnosis of Prostate Cancer," Urology, vol. 76, no. 5, pp. 1025-1033, 2010.

[2] P. Perrin, "Transrectal ultrasound for the diagnosis and staging of prostate cancer," Curr Opin Urol, vol. 2, no. 5, pp. 344-347, 1992.

[3] J. Coleman, R. Nascimento, and S. B. Solomon, "Advances in imaging for urologic procedures," Nat Clin Pract Urol, vol. 4, no. 9, pp. 498-504, 2007.

[4] A. Fenster and D. B. Downey, "Three-Dimensional Ultrasound Imaging," Annu Rev Biomed Eng, vol. 2, no. 1, pp. 457-475, 2000.

[5] S. Bae, P. Kim, and T. Song, "Ultrasonic sector imaging using plane wave synthetic focusing with a convex array transducer," J Acoust Soc Am, vol. 144, no. 5, pp. 2627-2644, 2018.

[6] J. H. Chang and T.-K. Song, "A New Synthetic Aperture Focusing Method to Suppress the Diffraction of Ultrasound," IEEE Trans Ultrason Ferroelectr Freq Control, vol. 58, no. 2, pp. 327-337, 2011.

[7] M.-H. Bae and M.-K. Jeong, "A study of syntheticaperture imaging with virtual source elements in B-mode ultrasound imaging systems," IEEE Trans Ultrason Ferroelectr Freq Control, vol. 47, no. 6, pp. 1510-1519, 2000.

[8] C. Kim, C. Yoon, J.-H. Park, Y. Lee, W. H. Kim, J. M. Chang, B. I. Choi, T.-K. Song, and Y.-M. Yoo, "Evaluation of Ultrasound Synthetic Aperture Imaging Using Bidirectional Pixel-Based Focusing: Preliminary Phantom and In Vivo Breast Study," IEEE Trans Biomed Eng, vol. 60, no. 10, pp. 2716$2724,2013$.

[9] J. A. Jensen, S. I. Nikolov, K. L. Gammelmark, and M. H. Pedersen, "Synthetic aperture ultrasound imaging," Ultrasonics, vol. 44, pp. e5-e15, 2006.

[10] S. I. Nikolov and J. A. Jensen, "3D synthetic aperture imaging using a virtual source element in the elevation plane," 2000 IEEE International Ultrasonics Symposium, vol. 2, pp. 1743-1747 vol.2, 2000.

[11] N. Bottenus, W. Long, H. K. Zhang, M. Jakovljevic, D. P. Bradway, E. M. Boctor, and G. E. Trahey, "Feasibility of 
Swept Synthetic Aperture Ultrasound Imaging," IEEE Trans Med Imaging, vol. 35, no. 7, pp. 1676-1685, 2016.

[12] M. H. Pedersen, K. L. Gammelmark, and J. A. Jensen, "In-vivo evaluation of convex array synthetic aperture imaging," Ultrasound Medicine Biology, vol. 33, no. 1, pp. 3747, 2007.

[13] H. Andresen, S. I. Nikolov, J. A. Jensen, H. Andresen, S. I. Nikolov, and J. A. Jensen, "Synthetic Aperture Focusing for a Single-Element Transducer Undergoing Helical Motion," IEEE Trans Ultrason Ferroelectr Freq Control, vol. 58, no. 5, pp. 935-943, 2011.

[14] J. Kortbek, Jø. A. Jensen, and K. Lø. Gammelmark, "Synthetic Aperture Sequential Beamforming," 2008 IEEE International Ultrasonics Symposium, pp. 966-969, 2008.

[15] H. Andresen, S. I. Nikolov, M. M. Pedersen, D. Buckton, and J. A. Jensen, "Three-Dimensional Synthetic Aperture Focusing Using a Rocking Convex Array Transducer," IEEE Trans Ultrason Ferroelectr Freq Control, vol. 57, no. 5, pp. 1051-1063, 2010.

[16] T. Lucas, I. Quidu, S. L. Bridal, and J. Gateau, "HighContrast and -Resolution 3-D Ultrasonography with a Clinical Linear Transducer Array Scanned in a Rotate-Translate Geometry," Appl Sci, vol. 11, no. 2, p. 493, 2021.

[17] S. Kang, J. Lee, and J. H. Chang, "Effectiveness of synthetic aperture focusing and coherence factor weighting for intravascular ultrasound imaging," Ultrasonics, vol. 113, p. 106364, 2021.

[18] J. S. Kim, J. G. Lee, J. H. Choi, B. H. Han, S. L. Yoon, H. Jung, T. K. Song, and J. Y. Lee, "A universal ultrasound diagnostic system developed to support urology and coloproctological applications," Biomed Eng Lett, vol. 9, no. 1, pp. 119-125, 2019.

[19] C. H. Frazier and W. D. O. Jr., "Synthetic aperture techniques with a virtual source element," IEEE Trans Ultrason Ferroelectr Freq Control, vol. 45, no. 1, pp. 196-207, 1998.

[20] J. A. Jensen and N. B. Svendsen, "Calculation of pressure fields from arbitrarily shaped, apodized, and excited ultrasound transducers," IEEE Trans Ultrason Ferroelectr Freq Control, vol. 39, no. 2, pp. 262-267, 1992.

[21] J. A. Jensen, "Field: A program for simulating ultrasound systems," Med Biol Eng Comput, vol. 34, pp. 351-353, Supplement 1, Part 1, 1996.

[22] Y. Lee, W. Y. Lee, C.-E. Lim, J. H. Chang, T.-K. Song, and Y. Yoo, "Compounded Direct Pixel Beamforming for Medical Ultrasound Imaging," IEEE Trans Ultrason Ferroelectr Freq Control, vol. 59, no. 3, pp. 573-582, 2012.

[23] Q. Hu, Z. X. Xie, Z. F. Wang, and Y. H. Liu, "Constructing NR-IQA Function Based on Product of Information Entropy and Contrast," 2008 Int Symposium Information Sci Eng, vol. 2, pp. 548-550, 2008.

[24] P.-H. Tsui, C.-K. Chen, W.-H. Kuo, K.-J. Chang, J. Fang, H.-Y. Ma, and D. Chou, "Small-window parametric imaging based on information entropy for ultrasound tissue characterization," Sci Rep-uk, vol. 7, no. 1, p. 41004, 2017.

[25] H. Ju, J. Kang, I. Song, and Y. Yoo, "A new multi-planar reconstruction method using voxel based beamforming for 3D ultrasound imaging," Proc SPIE, pp. 94124P-94124P-8, 2015.

[26] J. Kang, C. Yoon, J. Lee, S.-B. Kye, Y. Lee, J. H. Chang, G.-D. Kim, Y. Yoo, and T.-K. Song, "A System-on-Chip
Solution for Point-of-Care Ultrasound Imaging Systems: Architecture and ASIC Implementation," IEEE Trans Biomed Circ Systems, vol. 10, no. 2, pp. 412-423, Apr. 2016.

[27] Sewoong, Jeeun, Pilsu, Gunho, Eunji, Woojin, Minsuk, and Tai-Kyong, "Smartphone-Based Portable Ultrasound Imaging System: Prototype Implementation and Evaluation," 2015 IEEE International Ultrasonics Symposium, pp. 1-4.

[28] G.-D. Kim, C. Yoon, S.-B. Kye, Y. Lee, J. Kang, Y. Yoo, and T.-K. Song, "A Single FPGA-Based Portable Ultrasound Imaging System for Point-of-Care Applications," IEEE Trans Ultrason Ferroelectr Freq Control, vol. 59, no. 7, pp. 13861394, Jul. 2012.

[29] H. K. Zhang, F. Aalamifar, G. E. Trahey, and E. M. Boctor, "In vivo visualization of robotically implemented Synthetic Tracked Aperture Ultrasound (STRATUS) imaging system using curvilinear array," Proc SPIE, pp. 97901X97901X-7, 2016.

[30] L. V. Wang, "Multiscale photoacoustic microscopy and computed tomography," Nat Photonics, vol. 3, no. 9, pp. 503509, 2009.

[31] L. V. Wang and S. Hu, "Photoacoustic Tomography: In Vivo Imaging from Organelles to Organs," Science, vol. 335, no. 6075, pp. 1458-1462, 2012.

[32] J. Kang, E. Kim, G. R. Kim, C. Yoon, T. Song, and J. H. Chang, "Photoacoustic imaging of breast microcalcifications: A validation study with 3-dimensional ex vivo data and spectrophotometric measurement," J Biophotonics, vol. 8, no. $1-2$, pp. 71-80, 2015.

[33] J. Kang, J. H. Chang, S. M. Kim, H. J. Lee, H. Kim, B. C. Wilson, and T.-K. Song, "Real-time sentinel lymph node biopsy guidance using combined ultrasound, photoacoustic, fluorescence imaging: in vivo proof-of-principle and validation with nodal obstruction," Sci Rep-uk, vol. 7, no. 1, p. 45008, 2017.

[34] J. Kang, H. K. Zhang, S. D. Kadam, J. Fedorko, H. Valentine, A. P. Malla, P. Yan, M. M. Harraz, J. U. Kang, A. Rahmim, A. Gjedde, L. M. Loew, D. F. Wong, and E. M. Boctor, "Transcranial Recording of Electrophysiological Neural Activity in the Rodent Brain in vivo Using Functional Photoacoustic Imaging of Near-Infrared Voltage-Sensitive Dye," Front Neurosci-switz, vol. 13, p. 579, 2019.

[35] J. Kang, S. D. Kadam, J. S. Elmore, B. J. Sullivan, H. Valentine, A. P. Malla, M. M. Harraz, A. Rahmim, J. U. Kang, L. M. Loew, M. H. Baumann, A. A. Grace, A. Gjedde, E. M. Boctor, and D. F. Wong, "Transcranial photoacoustic imaging of NMDA-evoked focal circuit dynamics in the rat hippocampus," J Neural Eng, vol. 17, no. 2, p. 025001, 2020.

[36] W. Lesniak, Y. Yu, J. Kang, S. Boinapally, S. R. Banerjee, A. Lisok, A. Jabolonska, E. Boctor, and M. Pomper, "Dual Contrast Agents for Fluorescence and Photoacoustic Imaging: Evaluation in a Murine Model of Prostate Cancer," Nanoscale, 2021.

[37] H. K. Zhang, Y. Chen, J. Kang, A. Lisok, I. Minn, M. G. Pomper, and E. M. Boctor, "Prostate-specific membrane antigen-targeted photoacoustic imaging of prostate cancer in vivo," J Biophotonics, vol. 11, no. 9, p. e201800021, 2018. 